\title{
Subarachnoid hemorrhage: who dies, and why?
}

Hector Lantigua ${ }^{1 \dagger}$, Santiago Ortega-Gutierrez ${ }^{2 \dagger}$, J. Michael Schmidt ${ }^{1}$, Kiwon Lee $^{3}$, Neeraj Badjatia ${ }^{4}$, Sachin Agarwal ${ }^{1,5}$, Jan Claassen ${ }^{1,5}$, E. Sander Connolly ${ }^{5}$ and Stephan A. Mayer ${ }^{6^{*}}$

\begin{abstract}
Introduction: Subarachnoid hemorrhage $(\mathrm{SAH})$ is a devastating form of stroke. Causes and mechanisms of in-hospital death after SAH in the modern era of neurocritical care remain incompletely understood.

Methods: We studied 1200 consecutive SAH patients prospectively enrolled in the Columbia University SAH Outcomes Project between July 1996 and January 2009. Analysis was performed to identify predictors of in-hospital mortality.

Results: In-hospital mortality was $18 \%$ (216/1200): 3 \% for Hunt-Hess grade 1 or 2, $9 \%$ for grade 3, $24 \%$ for grade 4 , and $71 \%$ for grade 5 . The most common adjudicated primary causes of death or neurological devastation leading to withdrawal of support were direct effects of the primary hemorrhage (55\%), aneurysm rebleeding (17\%), and medical complications (15\%). Among those who died, brain death was declared in $42 \%, 50 \%$ were do-not-resuscitate at the time of cardiac death ( $86 \%$ of whom had life support actively withdrawn), and $8 \%$ died despite full support. Admission predictors of mortality were age, loss of consciousness at ictus, admission Glasgow Coma Scale score, large aneurysm size, Acute Physiology and Chronic Health Evaluation II (APACHE II) physiologic subscore, and Modified Fisher Scale score. Hospital complications that further increased the risk of dying in multivariable analysis included rebleeding, global cerebral edema, hypernatremia, clinical signs of brain stem herniation, hypotension of less than $90 \mathrm{~mm} \mathrm{Hg}$ treated with pressors, pulmonary edema, myocardial ischemia, and hepatic failure. Delayed cerebral ischemia, defined as deterioration or infarction from vasospasm, did not predict mortality.
\end{abstract}

Conclusion: Strategies directed toward minimizing early brain injury and aneurysm rebleeding, along with prevention and treatment of medical complication, hold the best promise for further reducing mortality after SAH.

\section{Introduction}

Subarachnoid hemorrhage (SAH) is devastating acute neurological disease that affects over 30,000 people every year in the United States [1-4]. Despite advances in medical and surgical management, SAH remains a major cause of premature mortality, accounting for $27 \%$ of all stroke-related potential years of life lost before the age of 65 [5]. In a 1985 study, it was reported that SAH carried a $43 \%$ risk of death immediately after ictus and a $57 \%$ mortality rate at 6 months [6]. A systematic review in 1997 evaluated cases-fatality rates from 1960 to 1992 and found a $0.9 \%$ decrease per year [7].

Well-established risk factors for mortality included poor clinical grade at presentation, older age, aneurysm

\footnotetext{
* Correspondence: stephan.mayer@mountsinai.org

${ }^{\dagger}$ Equal contributors

${ }^{6}$ Icahn School of Medicine at Mount Sinai, One Gustave L. Levy Place, Box 1522, New York, NY 10029-6574, USA

Full list of author information is available at the end of the article
}

rebleeding, large aneurysm size, and cerebral infarction from vasospasm [8]. The International Cooperative Aneurysm Study, conducted in the 1980s, pointed to vasospasm, direct effects of the primary hemorrhage, and rebleeding as the most frequent causes of mortality after SAH [9]. More recently, global cerebral edema, intraventricular hemorrhage, and medical complications have been identified as contributors to poor outcome after SAH [10-13]. In this study, our goal was to reevaluate the causes and mechanisms of in-hospital mortality after SAH in a large contemporary single-center cohort.

\section{Methods}

\section{Study population}

All spontaneous SAH patients admitted to the Neurological Intensive Care Unit at Columbia University Medical Center between July 1996 and January 2009 were offered enrollment in the Columbia University SAH
C Biomed Central

(c) 2015 Lantigua et al. Open Access This article is distributed under the terms of the Creative Commons Attribution 4.0 International License (http://creativecommons.org/licenses/by/4.0/), which permits unrestricted use, distribution, and reproduction in any medium, provided you give appropriate credit to the original author(s) and the source, provide a link to the Creative Commons license, and indicate if changes were made. The Creative Commons Public Domain Dedication waiver (http://creativecommons.org/publicdomain/zero/1.0/) applies to the data made available in this article, unless otherwise stated. 
Outcomes Project. Both patients with and those without a documented aneurysm were included in the analysis. The study was approved by the Columbia University Institutional Review Board, and in all cases written informed consent was obtained from the patient or a legally authorized representative. Patients were admitted to a dedicated neurological intensive care unit (ICU) and given treatment according to a standardized management protocol that has been described in detail previously; see Methods Supplement (Additional file 1) for details regarding inclusion and exclusion criteria, aneurysm management, and a description of our ICU management protocol and how it evolved over time [12, 14, 15].

\section{Clinical and radiological assessment}

Demographic data, social and medical history, and clinical features at onset were obtained shortly after admission. Neurological status was assessed with the Glasgow Coma Scale (GCS) [16] and the Hunt-Hess scale [17]. Physiologic stress was captured by using the Acute Physiology and Chronic Health Evaluation II (APACHE II) scale by calculating a physiological subscore after subtracting the GCS, age, and chronic health contributions to the total score [14]. Admission computed tomography (CT) scans, and those with significant interval changes during hospitalization, were evaluated by using the Modified Fisher Scale [15], Hijdra SAH sum score [18], and Intraventricular Hemorrhage Score [19] and for the presence of global cerebral edema [12]. During weekly meetings, a review of the entire hospital course was conducted to document and adjudicate important procedures, events, and complications according prespecified criteria [10]. Delayed cerebral ischemia (DCI) was defined as neurological deterioration, cerebral infarction, or both, when the cause was felt to be vasospasm after careful exclusion of other causes [20].

\section{Outcome assessment}

In-hospital mortality was used as the primary outcome for this analysis to allow for uniform evaluation of the causes, mode (i.e., brain versus cardiac), and level of support related to the dying process. The principal mechanism of death or neurological devastation leading to withdrawal of care was identified by adjudication of the study and clinical team after review of all pertinent clinical and radiographic findings. Primary causes of death were divided into eight categories based on the underlying pathophysiological mechanism: (1) direct effect of the primary hemorrhage, (2) aneurysm rebleeding, (3) cerebral infarction from vasospasm, (4) refractory cerebral edema leading to brain stem herniation, (5) hydrocephalus, (6) operative complications, (7) medical complications (e.g., fatal arrhythmia, pulmonary embolism, or multisystem organ failure who initially had a good neurological prognosis), and (8) other. Although different mechanisms could overlap in the same patient, only the adjudicated primary cause of death was considered.

\section{Witholding or withdrawal of support}

Do-not-resuscitate (DNR) status was systemically documented when instituted. Withdrawal of supportive care was defined as the cessation of intensive care support such as mechanical ventilation and the beginning of comfort care [21]. The treating team actively withdrew life support only at the direction of family members on the basis of dismal expected prospects for recovery and the known wishes of the patient on the basis of previous written or verbal statements.

\section{Statistical analysis}

Continuous variables were dichotomized to relevant clinical cut-points. Continuous variables were assessed for normality by using the Kolmogorov-Smirnov test. Normally distributed data were reported as a mean \pm standard deviation, and non-parametric data were reported as median and interquartile range (IQR). Univariable associations were tested by using chi-squared or Fisher's exact test for categorical variables, two-tailed $t$ test for normally distributed continuous variables, and Mann-Whitney $U$ test for non-normally distributed continuous variables. An initial multivariable analysis using logistic regression was performed to determine the relationship between premorbid demographic and admission clinical and radiographic variables and in-hospital mortality. For clinically intercorrelated variables that measure the same construct (e.g., admission clinical deficit measured by Hunt-Hess grade and GCS score), we selected the variable with the highest measure of association (odds ratio) and smallest $P$ value to be included in the final model. After construction of the baseline model for prediction of mortality, adjusted odds ratios for specific hospital complications were calculated by adding each of these factors individually to the baseline model to evaluate their unique contribution. Receiver operating characteristic (ROC) analysis was used to assess the ability of various models to predict in-hospital mortality. Nagelkerke's R-squared was used to estimate the percentage of the variance predicted by the combination of variables into the predicted model. Finally, a Hosmer-Lemeshow test was used to test goodness-of-fit of the each model. Residual statistics analysis was performed to evaluate the influence of isolated points into the model. Potential multicollinearity between the parameters of the final regression model was performed by calculating tolerance and variance inflation factor (VIF) coefficients. Significance was set at 0.05 for all analyses. All analyses were performed with commercially available statistical software (SPSS version 18.0; SPSS Inc., now part of IBM Corporation, Armonk, NY, USA). 


\section{Results}

\section{Overall mortality and mode of death}

Of the 1200 patients enrolled between July 1996 and January 2009, 216 (18 \%) died during hospitalization. Of those who died, $42 \%$ succumbed to brain death. Of those who died of cardiac death, $86 \%$ had a DNR order and $74 \%$ had life support actively withheld or withdrawn (Fig. 1). Mortality according to admission Hunt-Hess clinical grade is displayed in Table 1. Fifty-seven percent of patients underwent an aneurysm clipping procedure $(N=687), 19 \%(N=231)$ were coiled, and $24 \%$ underwent no procedure, due to either absence of an identifiable aneurysm $(N=148)$ or extremely poor clinical grade $(N=134)$. Mortality was higher among coiled $(14 \%)$ than clipped (7\%) patients. Median hospital lengths of stay were 14 (IQR 10-22) days for survivors and 5.5 (IQR 2$12)$ days among non-survivors $(P=0.001)$. Patients admitted in Hunt-Hess grade 3 to 5 condition represented over $90 \%$ of those who died.

\section{Timing of death}

Survival analysis stratified by mode of death and level of support is shown in Fig. 2. Thirty percent of deaths occurred within $48 \mathrm{~h}$ of admission, $56 \%$ had occurred by SAH day 7 , and $76 \%$ had occurred by SAH day 14 . The majority of brain dead patients $(74 \%, 67 / 90)$ died within 7 days of SAH, whereas death due to active withdrawal of support was more likely to occur after day 7 (55 \%, 51/ 93). Overall, the most common adjudicated primary causes of death (Fig. 3) were direct effect of the primary hemorrhage (55\%), aneurysm rebleeding (17\%), medical complications (15\%), cerebral edema (5\%), and DCI from vasospasm (5\%).

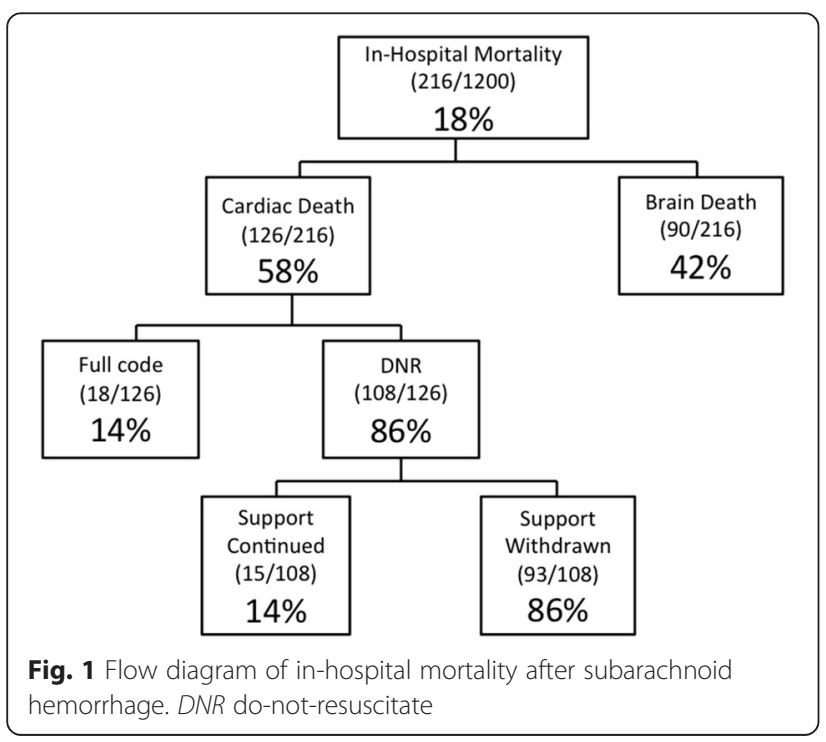

Table 1 Mortality according to admission Hunt-Hess grade

\begin{tabular}{lccc}
\hline Hunt-Hess grade & $\begin{array}{l}\text { Dead/Total, } \\
\text { number }\end{array}$ & $\begin{array}{l}\text { Proportion } \\
\text { of study } \\
\text { population, \% }\end{array}$ & $\begin{array}{l}\text { Mortality } \\
\text { rate, \% }\end{array}$ \\
\hline $\begin{array}{l}\text { 1. Mild headache } \\
\begin{array}{l}\text { 2. Severe headache or } \\
\text { cranial nerve deficit }\end{array}\end{array}$ & $12 / 342$ & 19.5 & 3.5 \\
$\begin{array}{l}\text { 3. Confusion, lethargy, } \\
\quad \text { or lateralized weakness }\end{array}$ & $30 / 3186$ & 15.5 & 3.2 \\
$\begin{array}{l}\text { 4. Stupor } \\
\text { 5. Coma }\end{array}$ & $41 / 173$ & 14.4 & 26.6 \\
Total & $127 / 180$ & 15.0 & 70.5 \\
\hline
\end{tabular}

\section{Temporal mortality trend}

Analysis of mortality over time according to admission clinical grade was performed by dividing the study population into four groups of 300 consecutively treated patients who received treatment over the course of about 3 years. Mortality remained consistently low for good-grade $\mathrm{SAH}$ patients (Hunt-Hess 1-3) over the 12-year period (Fig. 4), but there was a $20 \%$ absolute reduction in mortality among grade 5 patients between the first and second time epochs $\left(P=0.046, \chi^{2}\right.$ test for epoch 1 versus epochs $\left.2-4\right)$ and a similar $20 \%$ reduction among grade 4 patients between the third and fourth time epochs $\left(P=0.007, \chi^{2}\right.$ test for epochs 1-3 versus epoch 4$)$. The proportion of patients who underwent withdrawal of support, and the timing of withdrawal, did not change across time epochs.

\section{Admission predictors of mortality}

Twenty-two admission variables related to demographics, medical history, clinical features, imaging findings, and physiology were associated with in-hospital mortality in univariate analysis (Table 2). In a multivariable logistic regression analysis, age, loss of consciousness at ictus, APACHE II physiological subscore, admission GCS score, aneurysm size, and Modified Fisher Scale at admission were found to be the most important independent admission risk factors associated with in-hospital mortality (Nagelkerke R-squared $=0.497 ; \quad \mathrm{AUC}=0.90,95 \% \mathrm{CI}$ $0.87-0.93, P<0.0001$ ) (Table 3). No interactions or collinearity was found between any of the predictors included in the final model. Hosmer and Lemeshow test indicated adequacy of model fitting $(P=0.116)$. Both Cook's distance and Leverage residual coefficients were less than 1 for all individuals included in the study. Less than $5 \%$ of all standardized residuals were outside \pm 1.96 .

\section{Medical and neurological complications}

The vast majority of medical complications were overrepresented among patients who died (Table 4). After admission predictors of mortality were controlled for, 


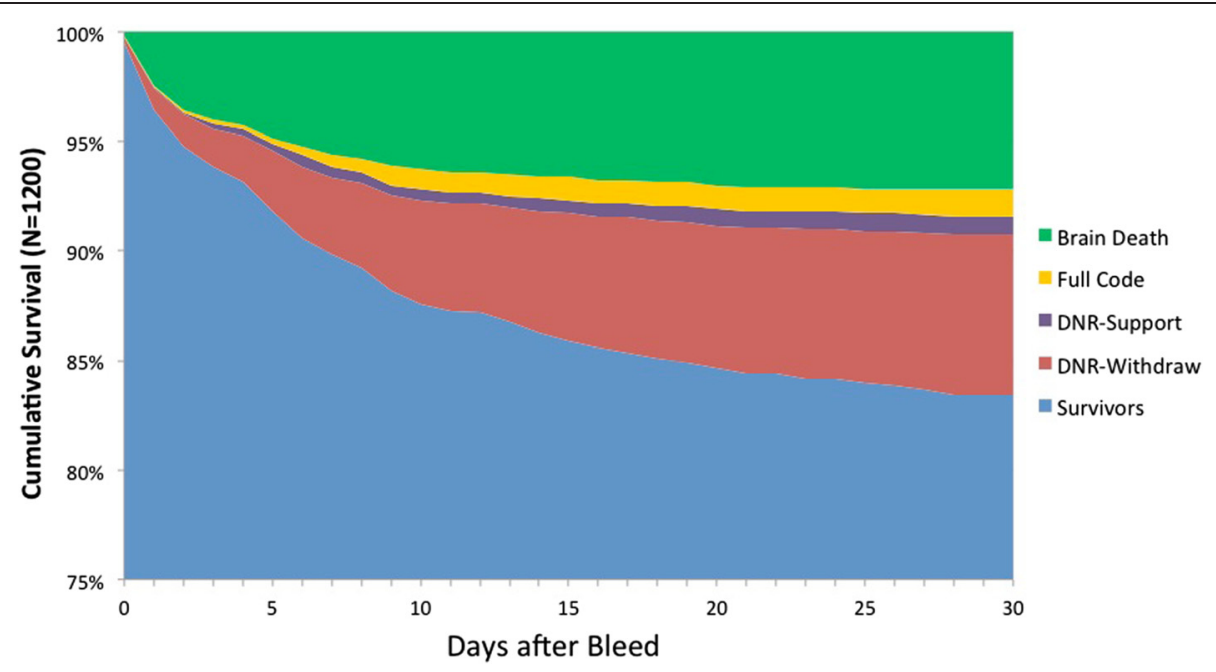

Fig. 2 Survival analysis stratified by mode of death and level of support during the first 2 weeks after SAH. An additional 17 patients (8 \% of those who died overall) died after SAH day 30 but prior to discharge. DNR do-not-resuscitate, SAH subarachnoid hemorrhage

complications that remained significantly associated with mortality included global cerebral edema, intracranial pressure (ICP) crisis or herniation events treated with bolus osmotherapy, hypotension (systolic blood pressure of less than $90 \mathrm{~mm} \mathrm{Hg}$ ) treated with pressors, congestive heart failure, aneurysm rebleeding, myocardial injury, and hepatic injury (Table 4). Of the $20 \%$ of the study population $(N=239)$ with diagnosed DCI, $92 \%$ received treatment with vasopressors and $38 \%$ with endovascular therapy in the form of intra-arterial verapamil (89\%) or balloon angioplasty (45\%) or both. These proportions did not change significantly over the four time epochs. DCI did not predict mortality.

\section{Discussion}

In this contemporary single-center study of 1200 cases of $\mathrm{SAH}$, hospital mortality was $18 \%$, which is on the low end of the range of $20-50 \%$ previously reported in the literature $[1,3-8,22,23]$. Although referral bias favoring transfer of good-grade patients can influence SAH mortality rates at tertiary care centers [24], HuntHess grades in our study population were broadly represented and consistent with those found in epidemiologic studies (Table 1) [4-7]. More likely, our results reflect the current trend of improvement in SAH outcomes that has occurred over the past two decades, with more aggressive aneurysm treatment protocols and advanced critical

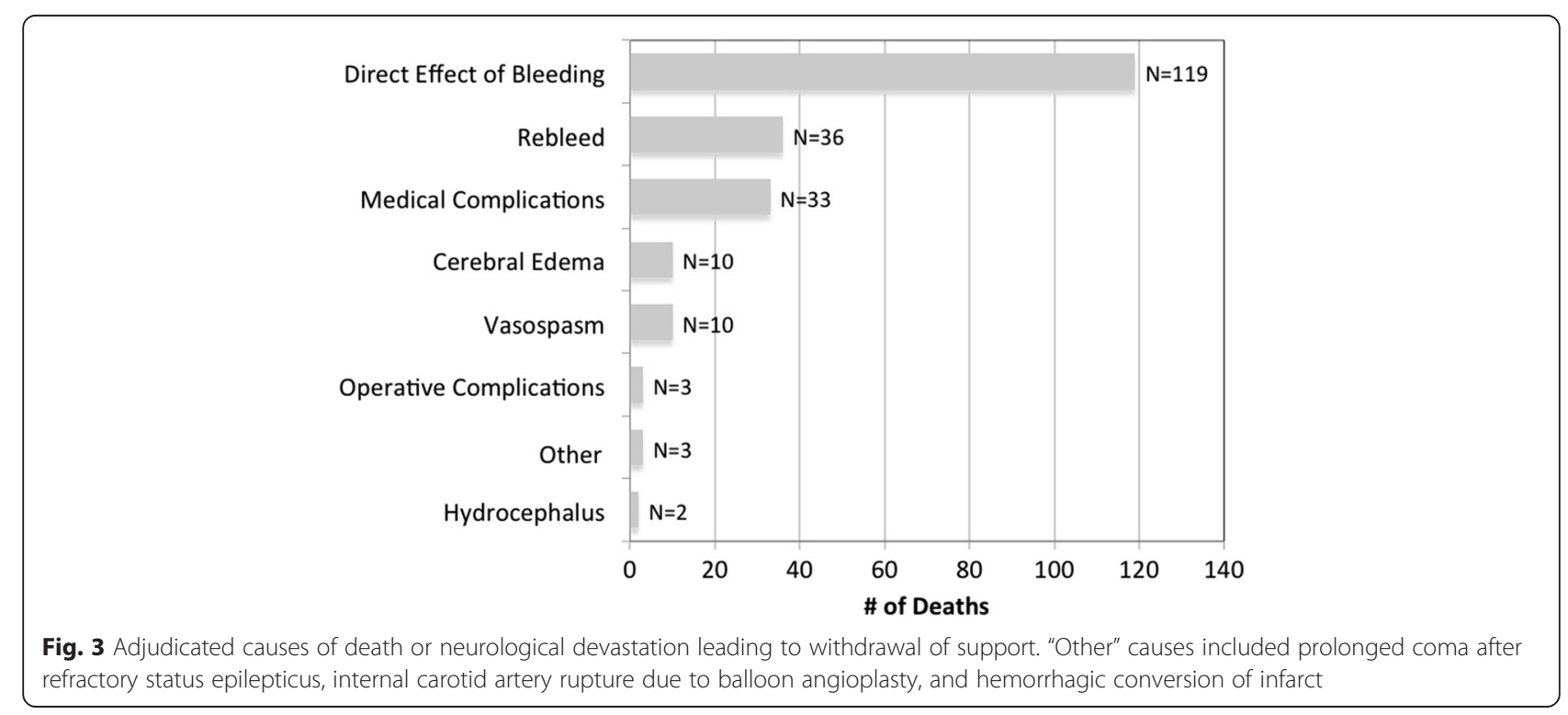




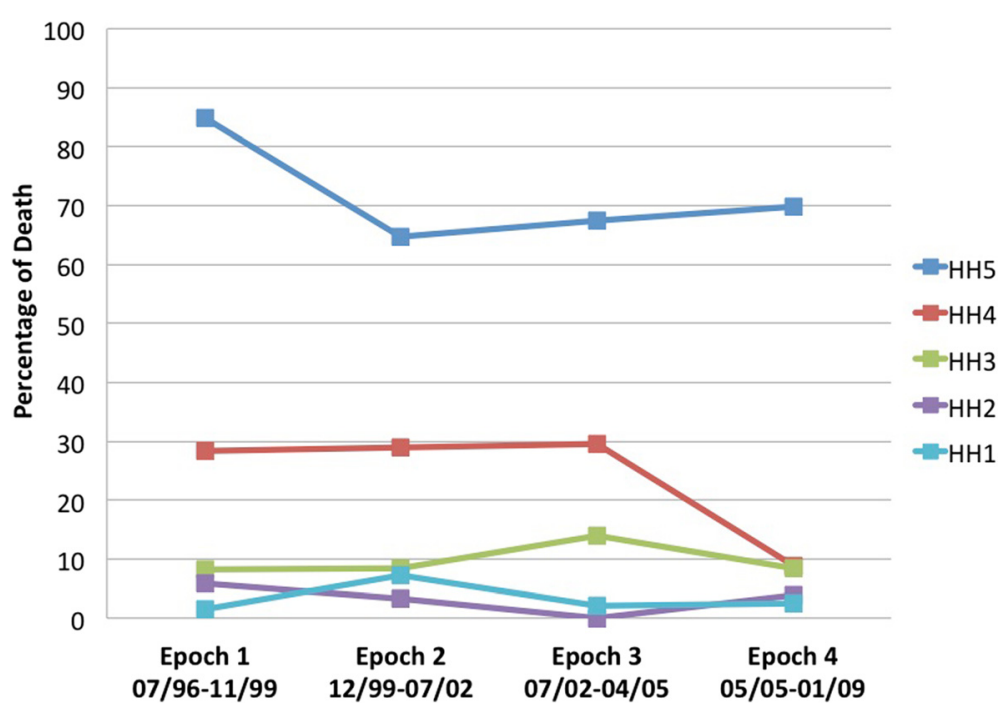

Fig. 4 Hospital mortality according to admission Hunt-Hess grade over the 12.5-year study period. Each time epoch represents 300 consecutive admissions. A dramatic fall in mortality was observed among grade 5 patients between epochs 1 and 2; a similar reduction occurred among grade 4 patients between epochs 3 and 4 . HH Hunt-Hess

care strategies directed at minimizing secondary injury $[3,7,8]$. This view is supported by the fact that mortality fell from approximately $85 \%$ to $70 \%$ among grade 5 patients early in the 12 -year study period and from $30 \%$ to $10 \%$ among grade 4 patients near the end of the study period (Fig. 4). SAH mortality has consistently been shown to be lower at high-volume as opposed to lowvolume centers $[3,25,26]$. As regionalization of complex stroke care at comprehensive stroke centers becomes more prevalent, risk-adjusted SAH mortality rates from specialized centers such as ours may serve as useful targets and benchmarks for evaluating and comparing quality of care between centers.

Forty-two percent of those who died in our study were pronounced brain dead. Most of these patients presented with loss of brainstem reflexes, had evidence of severe brain stem or diffuse cortical injury on CT, did not undergo angiography, and died quickly. Of the remaining patients, $50 \%$ were DNR ( $86 \%$ of whom had life support actively withdrawn) and only $8 \%$ died despite full medical support (Fig. 1). Two recent single-center studies of inhospital mortality after SAH reported similar rates of overall mortality (18-20\%) and withholding or withdrawal of support (50-76\%) compared with our study $[22,23]$. In general, we withdrew support from poor-grade patients at our center after an initial trial of aggressive surgical and critical care intervention. This protocol involved aneurysm repair (with a bias toward coiling) and external ventricular drainage whenever feasible and has come to include continuous electroencephalography, invasive brain multimodality monitoring, hypothermia for refractory intracranial pressure, and intra-arterial verapamil, balloon angioplasty, and intrathecal nicardipine for vasospasm as our practice has evolved [8].

$\mathrm{SAH}$ is a complicated disease that can involve multiple types of neurological injury and systemic organ dysfunction; it may be impossible to identify the precise contribution of each individual process to a patient's death. While acknowledging this problem, we sought to identify the "primary cause of death or neurological devastation leading to withdrawal of support" in weekly meetings of the research team after thorough review of all pertinent clinical and imaging data. The three most common adjudicated causes of death (Fig. 3) were direct effects of the primary hemorrhage (55\%), aneurysm rebleeding (17 \%), and medical complications (15\%). These findings suggest that, as of today, little has changed since a 1994 population-based study from the Greater Cincinnati region reported that initial and recurrent aneurysm rebleeding are the major causes of death after SAH [27].

Multivariable analysis of admission predictors (Table 3) and delayed complications confirms (Table 4) our adjudicated causes of death. Admission GCS score and Modified Fisher Scale are robust markers of the severity of the initial bleeding event, and both predicted inhospital mortality. Loss of consciousness at ictus is felt to reflect transient intracranial circulatory arrest, has been previously been linked to poor outcome and global cerebral edema at SAH onset $[8,28]$, and was also associated with mortality in our study. Global cerebral edema, a marker of diffuse brain injury that is usually present on admission CT, was associated with mortality when analyzed as a complication of SAH. Admission APACHE II physiological subscores capture extremes of 
Table 2 Baseline characteristics related to in-hospital mortality

\begin{tabular}{|c|c|c|c|c|}
\hline & Entire cohort & Survivors & Non-survivors & \\
\hline & $N=1200$ & $N=984$ & $N=216$ & P \\
\hline \multicolumn{5}{|l|}{ Demographics } \\
\hline Age & $55 \pm 15$ & $53 \pm 14$ & $60 \pm 16$ & 0.000 \\
\hline Female gender & $807(67)$ & $661(67)$ & $146(68)$ & 0.906 \\
\hline Non-white & $601(50)$ & $491(50)$ & $110(51)$ & 0.903 \\
\hline \multicolumn{5}{|l|}{ Medical history } \\
\hline Hypertension & $542(45)$ & $421(43)$ & $121(56)$ & 0.000 \\
\hline Diabetes & $89(7)$ & $76(8)$ & $13(6)$ & 0.916 \\
\hline Cigarette use in the last 6 months & $669(56)$ & $577(59)$ & $91(42)$ & 0.690 \\
\hline Alcohol use in the last 6 months & $652(54)$ & $587(60)$ & $65(30)$ & 0.000 \\
\hline Coronary artery disease & $52(4)$ & $34(4)$ & $18(8)$ & 0.000 \\
\hline Chronic obstructive pulmonary disease & $61(5)$ & $44(5)$ & $17(8)$ & 0.004 \\
\hline \multicolumn{5}{|l|}{ Admission clinical features } \\
\hline Loss of consciousness at ictus & $458(38)$ & $307(31)$ & $151(70)$ & 0.000 \\
\hline Tonic-clonic activity at ictus & $130(11)$ & $96(10)$ & $34(16)$ & 0.002 \\
\hline Sentinel headache & $203(17)$ & $162(17)$ & $41(20)$ & 0.032 \\
\hline Admission Glasgow Coma Scale score & $14(8-15)$ & $15(12-15)$ & $5(3-9)$ & 0.000 \\
\hline Admission Hunt-Hess grade & & & & 0.000 \\
\hline 1. Mild headache & $342(29)$ & $330(34)$ & $12(6)$ & \\
\hline 2. Severe headache & $186(16)$ & $180(18)$ & $6(3)$ & \\
\hline 3. Lethargy or confusion & $319(27)$ & $289(29)$ & $30(14)$ & \\
\hline 4. Stupor & $173(14)$ & $132(13)$ & $41(19)$ & \\
\hline 5. Coma & $180(15)$ & $53(5)$ & $127(59)$ & \\
\hline \multicolumn{5}{|l|}{ Admission imaging findings } \\
\hline Modified Fisher Score & & & & 0.000 \\
\hline 0. No blood & $66(6)$ & $61(6)$ & $5(2)$ & \\
\hline 1. Focal or diffuse thin SAH & $337(28)$ & $320(33)$ & $17(8)$ & \\
\hline 2. Focal or diffuse thin SAH with bilateral IVH & $106(9)$ & $82(8)$ & $24(11)$ & \\
\hline 3. Focal or diffuse thick SAH & $415(35)$ & $346(35)$ & $69(32)$ & \\
\hline 4. Focal or diffuse thick SAH with bilateral IVH & $246(21)$ & $148(15)$ & $98(45)$ & \\
\hline SAH sum score ${ }^{a}$ & $15(7-21)$ & $13(6-20)$ & $20(15-25)$ & 0.000 \\
\hline IVH sum score ${ }^{b}$ & $1(0-4)$ & $0(0-3)$ & $4(1-9)$ & 0.000 \\
\hline $\mathrm{ICH}$ present & $199(17)$ & $126(13)$ & $73(34)$ & 0.000 \\
\hline Global cerebral edema & $210(18)$ & $137(14)$ & $73(34)$ & 0.000 \\
\hline Hydrocephalus (bicaudate index >0.2) & $350(29)$ & $271(28)$ & $79(37)$ & 0.000 \\
\hline Aneurysm characteristics & & & & 0.000 \\
\hline Anterior cerebral artery & $318(26)$ & $275(28)$ & $43(20)$ & \\
\hline Internal carotid artery (includes P-comm) & $322(27)$ & $277(28)$ & $45(21)$ & \\
\hline Middle cerebral artery & $170(14)$ & $142(14)$ & $28(13)$ & \\
\hline Vertebrobasilar system & $183(15)$ & $153(16)$ & $30(14)$ & \\
\hline No aneurysm identified & $140(12)$ & $134(14)$ & $6(3)$ & \\
\hline No angiogram performed & $67(6)$ & $3(0.3)$ & $64(30)$ & \\
\hline Aneurysm diameter, mm & $7[5-10]$ & $7[5-10]$ & $10(6-15)$ & 0.000 \\
\hline Multiple aneurysms & $295(25)$ & $256(26)$ & $39(18)$ & 0.954 \\
\hline
\end{tabular}


Table 2 Baseline characteristics related to in-hospital mortality (Continued)

\begin{tabular}{|c|c|c|c|c|}
\hline \multicolumn{5}{|l|}{ Admission physiology } \\
\hline Systolic blood pressure, mm Hg & 156 [132-180] & 154 [132-178] & $164(131-196)$ & 0.003 \\
\hline Glucose level, mg/dl & 142 [118-176] & 136 [115-165] & $180[139-241]$ & 0.000 \\
\hline Troponin level, ng/dl & $0.26[0.02-0.3]$ & $0.1[0.02-0.3]$ & $0.3[0.02-1.6]$ & 0.000 \\
\hline APACHE physiological subscore ${ }^{c}$ & $5[3-8]$ & $5[3-7)]$ & $9[6-12]$ & 0.000 \\
\hline
\end{tabular}

Values are presented as mean \pm standard deviation, median [interquartile range], or number (percentage within each column).

SAH subarachnoid hemorrhage, IVH intraventricular hemorrhage, ICH intracerebral hemorrhage, APACHE Acute Physiology and Chronic Health Evaluation

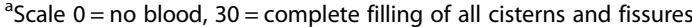

${ }^{\mathrm{b}} \mathrm{Scale} 0=$ no IVH, 12 = complete filling of all ventricles

${ }^{\mathrm{c} S} \mathrm{~S}$ ale $0=$ no physiological derangement, $44=$ maximal physiological derangeme

blood pressure, hypoxia, fever, and other signs of systemic inflammation; their association with mortality suggests that these derangements may aggravate early brain injury [14]. Multivariable analysis confirmed aneurysm rebleeding as an important cause of in-hospital death, with a surprisingly high event rate of $10 \%$ despite our policy of performing aneurysm repair as quickly as possible [29]. As we have reported previously, $73 \%$ of in-hospital rebleeding events in our patient population occurred within $72 \mathrm{~h}$ of the index hemorrhage precipitating admission; the main risk factors for rebleeding were poor Hunt-Hess grade and large aneurysm size [29]. Non-modifiable admission predictors of mortality included age, pre-existing hypertension, and large aneurysm size, confirming the results of many other studies [10, 12, 14, 15, 30, 31].

Medical complications directly accounted for $15 \%$ of in-hospital deaths in our study, which is lower than the $23 \%$ proportion of deaths attributed to medical complications in the Cooperative Aneurysm Study [32]. This discrepancy may be explained by the fact that poorgrade patients were under-represented in the Cooperative Study. We previously reported in a subset of patients $(N=580)$ included our study cohort that fever of more than $38.3{ }^{\circ} \mathrm{C}$, hyperglycemia of more than $200 \mathrm{mg} /$ $\mathrm{dl}$, and anemia treated with transfusion were significant predictors of either death or moderate-to-severe disability 3 months after SAH [10]. We did not find an independent association between these complications and in-hospital mortality in the present analysis, despite

Table 3 Admission predictors of in-hospital mortality: multivariable analysis

\begin{tabular}{lllc}
\hline Variable & OR & $95 \% \mathrm{Cl}$ & $P$ value \\
\hline Age, years & 1.04 & $1.0-1.1$ & 0.00 \\
Loss of consciousness at ictus & 1.99 & $1.3-3.2$ & 0.03 \\
Admission GCS score & 0.77 & $0.7-0.8$ & 0.00 \\
Aneurysm size, per mm & 1.10 & $1.1-1.2$ & 0.00 \\
APACHE II physiologic subscore & 1.08 & $1.0-1.1$ & 0.02 \\
Modified Fisher Scale score & 1.25 & $1.0-1.5$ & 0.03 \\
\hline
\end{tabular}

OR odds ratio, $\mathrm{Cl}$ confidence interval, GCS Glasgow Coma Scale, APACHE II Acute Physiology and Chronic Health Evaluation II confirming that these long-term associations with poor functional outcome persist (unpublished data). This suggests that prolonged fever, hyperglycemia, and anemia contribute more directly to disability than to mortality per se, perhaps by contributing to neurological dysfunction and deconditioning.

Complications associated with refractory cerebral edema and cardiopulmonary dysfunction were both broadly associated with in-hospital death. In addition to global cerebral edema (which affected $26 \%$ overall), ICP crisis or neurological worsening treated with bolus osmotherapy (22\%), hypernatremia (21\%), and clinical signs of brain stem herniation (14\%) were all associated with mortality after admission predictors were controlled for (Table 4). The association with hypernatremia primarily reflects the effect of osmotherapy since diabetes insipidus was formally diagnosed in only $4 \%$ of our study population [10]. In our view, ICP, herniation, and brain edema complications generally constitute a downstream effect of severe diffuse early brain injury. Specific cardiopulmonary complications associated with mortality included hypotension of less than $90 \mathrm{~mm} \mathrm{Hg}$ treated with pressors (which affected $24 \%$ overall), congestive heart failure (8\%), and nonneurogenic myocardial ischemia (7\%), which was defined as acute or delayed troponin elevation possibly or probably due to coronary ischemia. The association of troponin elevation with stunned myocardium, hypotension, pulmonary edema, and poor outcome after SAH is well described [11,33,34] and supports multimodality monitoring data implicating hypoxia and hypotension as important causes of secondary brain injury [35].

A robust association between vasospasm and in-hospital mortality is notably absent in the present study. DCI from vasospasm was the adjudicated primary cause of death in $5 \%$ of the cases, and DCI analyzed as a complication failed to independently predict death after admission risk factors were taken into account. By contrast, symptomatic vasospasm was the leading primary cause of death or disability among patients receiving treatment in the $1990 \mathrm{Co}-$ operative Aneurysm Study, accounting for $28 \%$ of all deaths [9]. This change most likely reflects the impact of interventions that have since reduced the likelihood of 
Table 4 Relationship of medical and neurological complications to in-hospital mortality

\begin{tabular}{|c|c|c|c|c|c|c|c|c|}
\hline & \multirow[b]{2}{*}{ Survivors } & \multirow[b]{2}{*}{ Non-survivors } & \multicolumn{3}{|c|}{ Univariate/Unadjusted } & \multicolumn{3}{|c|}{ Multivariate/Adjusted $^{a}$} \\
\hline & & & $\mathrm{OR}$ & $95 \% \mathrm{Cl}$ & $P$ value & $\mathrm{OR}$ & $95 \% \mathrm{Cl}$ & $P$ value \\
\hline Fever $>101.5 \mathrm{~F}$ & $480(49)$ & $137(63)$ & 1.9 & $1.4-2.6$ & 0.000 & 0.8 & $0.5-1.3$ & 0.35 \\
\hline Hyperglycemia, >200 mg/dl & $408(42)$ & $143(66)$ & 2.9 & $2.1-4.0$ & 0.000 & 1.1 & $0.7-1.8$ & 0.76 \\
\hline Hydrocephalus requiring EVD or VPS & $289(29)$ & $142(66)$ & 4.7 & $3.5-6.5$ & 0.417 & 1.4 & $0.9-2.2$ & 0.15 \\
\hline Anemia requiring transfusion & $319(32)$ & $75(35)$ & 1.1 & $0.8-1.6$ & 0.000 & 0.7 & $0.45-1.0$ & 0.10 \\
\hline Global cerebral edema & $205(21)$ & $107(50)$ & 3.7 & $2.8-5.2$ & 0.000 & 1.8 & $1.1-2.9$ & 0.02 \\
\hline New infarct on CT scan & $233(24)$ & $78(36)$ & 2.0 & $1.5-2.8$ & 0.000 & 0.7 & $0.4-1.1$ & 0.09 \\
\hline ICP crisis or herniation ${ }^{\mathrm{a}}$ & $153(16)$ & $106(49)$ & 5.7 & $4.0-7.9$ & 0.000 & 2.5 & $1.4-3.7$ & 0.00 \\
\hline Hypotension, $<90$ mm Hg ${ }^{b}$ & $160(16)$ & $122(57)$ & 6.7 & $4.9-9.2$ & 0.000 & 3.4 & $2.2-5.3$ & 0.00 \\
\hline Pneumonia & $191(19)$ & $67(31)$ & 1.9 & $1.4-2.7$ & 0.000 & 0.6 & $0.4-1.0$ & 0.06 \\
\hline Hypernatremia, >150 mEq/l & $151(15)$ & $99(46)$ & 5.1 & $3.6-7.1$ & 0.000 & 2.1 & $1.3-3.4$ & 0.00 \\
\hline Urinary tract infection & $226(23)$ & $13(6)$ & 0.2 & $0.1-0.4$ & 0.881 & 0.1 & $0.0-0.2$ & 0.00 \\
\hline Clinical deterioration from vasospasm & $170(17)$ & $36(17)$ & 0.9 & $0.7-1.4$ & 0.000 & 0.9 & $0.6-1.6$ & 0.81 \\
\hline Pulmonary edema & $137(14)$ & $68(32)$ & 2.9 & $2.0-4.1$ & 0.000 & 1.3 & $0.8-2.0$ & 0.33 \\
\hline Herniation & $62(6)$ & $106(50)$ & 15.3 & $10.5-22.1$ & 0.052 & 8.3 & $4.9-14.3$ & 0.00 \\
\hline Hyponatremia, <130 mEq/l & $140(14)$ & $20(9)$ & 0.6 & $0.4-1.0$ & 0.010 & 0.5 & $0.3-0.96$ & 0.04 \\
\hline Sepsis/Bacteremia & $91(9)$ & $32(15)$ & 1.8 & $1.1-2.7$ & 0.000 & 1.4 & $0.79-2.4$ & 0.26 \\
\hline Arrhythmia & $71(7)$ & $49(23)$ & 3.7 & $2.5-5.6$ & 0.000 & 1.6 & $0.9-2.8$ & 0.09 \\
\hline Aneurysm rebleeding & $54(6)$ & $64(30)$ & 6.9 & $4.6-10.4$ & 0.000 & 3.5 & $1.9-5.9$ & 0.00 \\
\hline Congestive heart failure & $62(6)$ & $39(18)$ & 3.3 & $2.1-5.1$ & 0.001 & 2.2 & $1.3-3.8$ & 0.00 \\
\hline Seizures & $55(6)$ & $25(12)$ & 2.3 & $1.4-3.8$ & 0.068 & 1.6 & $0.8-3.0$ & 0.20 \\
\hline \multicolumn{9}{|l|}{ Delayed cerebral ischemia } \\
\hline Symptomatic vasospasm without infarct & $104(11)$ & $14(7)$ & 0.6 & $0.3-1.0$ & 0.095 & 0.5 & $0.3-1.0$ & 0.06 \\
\hline Symptomatic vasospasm with infarct & $64(7)$ & $21(10)$ & 1.5 & $0.9-2.6$ & 0.004 & 1.9 & $1.0-3.7$ & 0.07 \\
\hline No symptomatic vasospasm with infarct & $23(2)$ & $13(6)$ & 2.7 & $1.3-5.4$ & 0.000 & 2.2 & $0.8-5.8$ & 0.13 \\
\hline Non-neurogenic myocardial ischemia & $46(5)$ & $43(20)$ & 5.1 & $3.3-8.1$ & 0.000 & 2.8 & $1.6-5.1$ & 0.00 \\
\hline Hepatic injury, AST or ALT >200 mg/dl & $24(2)$ & $22(10)$ & 4.6 & $2.5-8.4$ & 0.000 & 2.5 & $1.2-5.3$ & 0.01 \\
\hline Gl bleeding requiring transfusion & $19(2)$ & $14(7)$ & 3.5 & $1.7-7.1$ & 0.000 & 1.7 & $0.7-4.3$ & 0.27 \\
\hline
\end{tabular}

Values are presented as number (percentage)

$O R$ odds ratio, $C l$ confidence interval, EVD external ventricular drain, VPS ventriculoperitoneal shunt, $C T$ computed tomography, ICP intracranial pressure, $A S T$ aspartate transaminase, $A L T$ alanine transaminase, GI gastrointestinal

Adjusted ORs for in-hospital mortality are calculated individually for each complication after adjustment for admission mortality predictors (Table 3). See reference [9] for complete definitions

${ }^{a}$ Treated with osmotherapy

${ }^{\mathrm{b}}$ Treated with vasopressors

devastating brain infarction leading to withdrawal of support, including deployment of pharmacologic hemodynamic augmentation and intra-arterial therapy at the first sign of symptomatic vasospasm [8]. Even with aggressive management, there is ample evidence that brain infarction from DCI continues to be an important cause of cognitive impairment and disability after SAH $[36,37]$.

This study has several important limitations. First, these data were collected from a single highly specialized center and this may limit generalizability. Second, our overall management approach evolved over the 12-year enrollment period, as we eventually moved toward routinely offering of a 1-week trial of surgical or endovascular aneurysm repair and aggressive ICU support for all but the most moribund cases. Our attempt to identify an adjudicated "primary cause of death or neurological devastation leading to withdrawal of support" doubtlessly resulted in over-simplification in some cases, but we felt that trying to assign weights to multiple contributing factors would be even more arbitrary. Although we used pre-defined criteria for identifying medical complications, varying application of these criteria and observer bias by study team members that changed over the years may have affected the accuracy and consistency of how various findings and events were coded. We identified temporal trends showing lower mortality in Hunt-Hess grade 4 and 5 patients 
over time. In future studies, we will attempt to elucidate possible explanations for these improvements in outcome. Finally, as a consequence of restricting the scope of our analysis to in-hospital mortality, we did not analyze longterm survival or functional and quality-of-life outcomes after discharge.

\section{Conclusions}

Our findings suggest that strategies directed toward minimizing early brain injury and aneurysm rebleeding, along with prevention and treatment of cardiovascular complications, hold the best promise for further reducing mortality after SAH. Neuroprotection directed at minimizing the initial catastrophic diffuse brain insult and interventions designed to stabilize acute physiological derangements are promising targets for therapy [8]. Early use of brain multimodality monitoring may aid in the development of strategies directed at minimizing metabolic crisis, brain tissue hypoxia, spreading depression, and other potential mediators of early brain injury $[13,35]$. Routine use of anti-fibrinolytic therapy within $72 \mathrm{~h}$ of onset to minimize the risk of early rebleeding has been shown to be effective in a clinical trial and a single-center implementation study [38, 39]. Trials of acute sympatholytic therapy and advanced cardiovascular monitoring aimed at minimizing myocardial catecholamine toxicity and the frequency and severity of major adverse cardiovascular events deserve further study.

Premature withholding or withdrawal of support on the basis of self-fulfilling prophecies without offering a genuine trial of aggressive early resuscitation may be a powerful determinant of mortality after SAH [23]. We feel that, whenever possible, an initial trial of full support, at a minimum including aneurysm repair and ventriculostomy placement, should be offered to all potentially viable poorgrade $\mathrm{SAH}$ patients if consistent with their previously stated wishes. We have previously noted that an early aggressive trial of ICU support may actually improve satisfaction and subsequently make it easier for families to change the goal of care to comfort, knowing full well that everything possible was done $[8,40]$. Finally, our findings provide a benchmark for evaluating SAH treatment centers and demonstrate that reductions in mortality from this devastating disease are possible.

\section{Key messages}

- In-hospital mortality in this single-center cohort study of patients with SAH was $18 \%$

- $42 \%$ died of brain death, $50 \%$ were DNR, and only $8 \%$ died despite full medical support

- Direct effects of severe hemorrhage, rebleeding, and medical complications were the most common causes of death
- Delayed cerebral ischemia from vasospasm did not predict mortality

\section{Additional file}

Additional file 1: Methods Supplement. This provides details regarding inclusion and exclusion criteria, aneurysm management, and a description of our intensive care unit management protocol and how it evolved over time. (DOCX $26 \mathrm{~kb}$ )

\section{Abbreviations}

APACHE: Acute Physiology and Chronic Health Evaluation; CT: Computed tomography; DCl: Delayed cerebral ischemia; DNR: Do not resuscitate; GCS: Glasgow Coma Scale; ICP: Intracranial pressure; ICU: Intensive care unit; IQR: interquartile range; SAH: Subarachnoid hemorrhage.

\section{Competing interests}

SAM has received consulting fees from Actelion Pharmaceuticals (Allschwil, Switzerland) and Edge Therapeutics (Berkeley Heights, NJ, USA). The other authors declare that they have no competing interests.

\section{Authors' contributions}

HL performed data management, conducted the data analysis, interpreted the findings, and drafted the manuscript. SO-G performed data collection, interpreted the data, and assisted in drafting the manuscript. JMS maintained the project database, performed the statistical analyses, interpreted the data, and critically revised the manuscript for important intellectual content. KL, $\mathrm{NB}, \mathrm{SA}$, and JC collected and interpreted the data and critically revised the manuscript for important intellectual content. ESC assisted in the design of the study and data analysis, adjudicated adverse events, and critically revised the manuscript for important intellectual content. SAM designed the study, collected and interpreted the data, and drafted the manuscript. All authors have read and approved the final manuscript and agree to be accountable for all aspects of the work.

\section{Acknowledgments}

SO-G received a 2012 SPOTRIAS fellowship funded by National Institutes of Neurological Diseases and Stroke (NINDS P50 NS049060). SAM was supported by a Grant-in-Aid from the American Heart Association (\#9750432 N). This publication was supported by the National Center for Advancing Translational Sciences, National Institutes of Health (through grant UL1 TR000040), formerly the National Center for Research Resources (grant UL1 RR024156).

\section{Author details}

${ }^{1}$ Department of Neurology, Columbia University College of Physicians and Surgeons, 177 Fort Washington Ave, New York, NY 10032, USA. ${ }^{2}$ Department of Neurology, University of lowa, 200 Hawkins Drive, lowa City, IA 52242, USA. ${ }^{3}$ The University of Texas at Houston, 6431 Fannin St., Houston, TX 77030, USA. ${ }^{4}$ The University of Maryland, 22 South Greene St., Baltimore, MD 21201, USA. ${ }^{5}$ Department of Neurosurgery, Columbia University College of Physicians and Surgeons, 710 West 168th St., New York, NY 10032, USA. ${ }^{6}$ Icahn School of Medicine at Mount Sinai, One Gustave L. Levy Place, Box 1522, New York, NY 10029-6574, USA.

Received: 12 June 2015 Accepted: 17 August 2015

Published online: 31 August 2015

\section{References}

1. Longstreth Jr WT, Nelson LM, Koepsell TD, van Belle G. Clinical course of spontaneous subarachnoid hemorrhage: a population-based study in king county, Washington. Neurology. 1993;43:712-8.

2. Sudlow CL, Warlow CP. Comparable studies of the incidence of stroke and its pathological types: results from an international collaboration. International stroke incidence collaboration. Stroke. 1997;28:491-9.

3. Rincon F, Rosenwasser RH, Dumont A. The epidemiology of admissions of non-traumatic subarachnoid hemorrhage in the United States. Neurosurg. 2013;73:217-22. 
4. Go AS, Mozaffarian D, Roger VL, Benjamin EJ, Berry JD, Borden WB, et al. Heart disease and stroke statistics-2013 update: a report from the American Heart Association. Circulation. 2013;127:e6-245.

5. Johnston SC, Selvin S, Gress DR. The burden, trends, and demographics of mortality from subarachnoid hemorrhage. Neurology. 1998;50:1413-8.

6. Bonita R, Thomson S. Subarachnoid hemorrhage: epidemiology, diagnosis, management, and outcome. Stroke. 1985;16:591-4.

7. Stegmayr B, Eriksson M, Asplund K. Declining mortality from subarachnoid hemorrhage: changes in incidence and case fatality from 1985 through 2000. Stroke. 2004:35:2059-63.

8. Komotar RJ, Schmidt JM, Starke RM, Claassen J, Wartenberg KE, Lee K, et al. Resuscitation and critical care of poor-grade subarachnoid hemorrhage. Neurosurgery. 2009;64:397-410.

9. Kassell NF, Torner JC, Haley Jr EC, Jane JA, Adams HP, Kongable GL. The international cooperative study on the timing of aneurysm surgery. Part 1: Overall management results. J Neurosurg. 1990;73:18-36.

10. Wartenberg KE, Schmidt JM, Claassen J, Temes RE, Frontera JA, Ostapkovich $\mathrm{N}$, et al. Impact of medical complications on outcome after subarachnoid hemorrhage. Critical Care Med. 2006;34:617-23.

11. van der Bilt IA, Hasan D, Vandertop WP, Wilde AA, Algra A, Visser FC, et al. Impact of cardiac complications on outcome after aneurysmal subarachnoid hemorrhage: a meta-analysis. Neurology. 2009;72:635-42.

12. Claassen J, Carhuapoma JR, Kreiter KT, Du EY, Connolly ES, Mayer SA. Global cerebral edema after subarachnoid hemorrhage: frequency, predictors, and impact on outcome. Stroke. 2002;33:1225-32.

13. Helbok R, Ko SB, Schmidt JM, Kurtz P, Fernandez L, Choi HA, et al. Global cerebral edema and brain metabolism after subarachnoid hemorrhage. Stroke. 2011:42:1534-9.

14. Claassen J, Vu A, Kreiter KT, Kowalski RG, Du EY, Ostapkovich N, et al. Effect of acute physiologic derangements on outcome after subarachnoid hemorrhage. Critical Care Med. 2004;32:832-8.

15. Claassen J, Bernardini GL, Kreiter K, Bates J, Du YE, Copeland D, et al. Effect of cisternal and ventricular blood on risk of delayed cerebral ischemia after subarachnoid hemorrhage: The fisher scale revisited. Stroke. 2001;32:2012-20.

16. Teasdale G, Jennett B. Assessment of coma and impaired consciousness. A practical scale. Lancet. 1974;2:81-4.

17. Hunt WE, Hess RM. Surgical risk as related to time of intervention in the repair of intracranial aneurysms. J Neurosurg. 1968;28:14-20.

18. Hijdra A, Brouwers PJ, Vermeulen M, van Gijn J. Grading the amount of blood on computed tomograms after subarachnoid hemorrhage. Stroke. 1990;21:1156-61

19. Brouwers PJ, Dippel DW, Vermeulen M, Lindsay KW, Hasan D, van Gijn J. Amount of blood on computed tomography as an independent predictor after aneurysm rupture. Stroke. 1993;24:809-14.

20. Frontera JA, Fernandez A, Schmidt JM, Claassen J, Wartenberg KE, Badjatia $\mathrm{N}$, et al. Defining vasospasm after subarachnoid hemorrhage: What is the most clinically relevant definition? Stroke. 2009;40:1963-8.

21. Matchett SC, Castaldo J, Wasser TE, Baker K, Mathiesen C, Rodgers J. Predicting mortality after intracerebral hemorrhage: comparison of scoring systems and influence of withdrawal of care. J Stroke Cerebrovasc Dis. 2006;15:144-50.

22. Lee VH, Ouyang B, John S, Conners JJ, Garg R, Bleck TP, et al. Risk stratification for the in-hospital mortality after subarachnoid hemorrhage: the HAIR score. Neurocrit Care. 2014;21:14-9.

23. Kowalski RG, Chang TR, Carhuapoma JR, Tamargo R, Naval NS. Withdrawal of technological life support following subarachnoid hemorrhage. Neurocrit Care. 2013;19:269-75.

24. Whisnant JP, Sacco SE, O'Fallon WM, Fode NC, Sundt TM. Referral bias in aneurysmal subarachnoid hemorrhage. J Neurosurg. 1993;78:726-32

25. Solomon RA, Mayer SA, Tarmey J. Relationship between the volume of craniotomies for cerebral aneurysm performed at New York State hospitals and in-hospital mortality. Stroke. 1996;27:13-7.

26. Berman MF, Solomon RA, Mayer SA, Yung P. Impact of demographic and hospital-related factors on outcome following treatment for cerebral aneurysms. Stroke. 2003;34:2200-7.

27. Broderick J, Brott TG, Duldner JE, Tomsick T, Leach A. Initial and recurrent rebleeding are the major causes of death following subarachnoid hemorrhage. Stroke. 1994;25:1342-7.

28. Hop JW, Rinkel GJE, Algra A, van Gijn J. Initial loss of consciousness and risk of delayed cerebral ischemia after aneurysmal subarachnoid hemorrhage. Stroke. 1999;30:2268-71.
29. Naidech AM, Janjua N, Kowalski RG, Kreiter KT, Ostapkovich ND, Fitzsimmons B-F, et al. Predictors and impact of aneurysm rebleeding after subarachnoid hemorrhage. Arch Neurol. 2005;62:410-6.

30. Eskesen V, Rosenorn J, Schmidt K, Ronde F. Pre-existing arterial hypertension in subarachnoid haemorrhage: an unfavourable prognostic factor. $\mathrm{Br} J$ Neurosurg. 1987;1:455-61.

31. Juvela S, Siironen J, Kuhmonen J. Hyperglycemia, excess weight, and history of hypertension as risk factors for poor outcome and cerebral infarction after aneurysmal subarachnoid hemorrhage. J Neurosurg. 2005;102:998-1003.

32. Solenski NJ, Haley Jr EC, Kassell NF, Kongable G, Germanson T, Truskowski L, et al. Medical complications of aneurysmal subarachnoid hemorrhage: a report of the multicenter, cooperative aneurysm study. Crit Care Med. 1995;23:1007-17.

33. Naidech AM, Kreiter KT, Janjua N, Ostapkovich ND, Parra A, Commichau C, et al. Cardiac troponin elevation, cardiovascular morbidity, and outcome after subarachnoid hemorrhage. Circulation. 2005;112:2851-6.

34. Zaroff JG, Rordorf GA, Newell J, Ogilvy CS. Cardiac outcome in patients with subarachnoid hemorrhage and electrocardiographic abnormalities. Neurosurg. 1999;44:34-9.

35. Schmidt JM, Ko SB, Helbok R, Kurtz P, Stuart RM, Presciutti M, et al. Cerebra perfusion pressure thresholds for brain tissue hypoxia and metabolic crisis after poor-grade subarachnoid hemorrhage. Stroke. 2011;42:1531-6.

36. Kreiter KT, Copeland DL, Bernardini GL, Bates J, Peery S, Claassen J, et al. Predictors of cognitive dysfunction after subarachnoid hemorrhage. Stroke. 2002;33:200-9.

37. Etminan N, Vergouwen MD, Macdonald RL. Angiographic vasospasm versus cerebral infarction as outcome measures after aneurysmal subarachnoid hemorrhage. Acta Neurochirurgica. 2013;115:33-40.

38. Hillman J, Fridriksson S, Nilsson O, Yu Z, Saveland H, Jakobsson KE. Immediate administration of tranexamic acid and reduced incidence of early rebleeding after aneurysmal subarachnoid hemorrhage: a prospective randomized study. J Neurosurg. 2002;97:771-8.

39. Starke RM, Kim GH, Fernandez A, Komotar RJ, Hickman ZL, Otten ML, et al. Impact of a protocol for acute antifibrinolytic therapy on aneurysm rebleeding after subarachnoid hemorrhage. Stroke. 2008;39:2617-21.

40. Mayer SA, Kossoff SB. Withdrawal of life support in the neurological intensive care unit. Neurology. 1999;52:1602-9.

\section{Submit your next manuscript to BioMed Central and take full advantage of:}

- Convenient online submission

- Thorough peer review

- No space constraints or color figure charges

- Immediate publication on acceptance

- Inclusion in PubMed, CAS, Scopus and Google Scholar

- Research which is freely available for redistribution 